\title{
LATTICE BOLTZMANN STUDY OF ELECTROHYDRODYNAMIC DROP DEFORMATION WITH LARGE DENSITY RATIO
}

\author{
ZHI-TAO LI, GAO-JIN LI, HAI-BO HUANG and XI-YUN LU \\ Department of Modern Mechanics \\ University of Science and Technology of China \\ Hefei, Anhui 230026, China
}

Received 27 January 2011

Accepted 20 June 2011

\begin{abstract}
The lattice Boltzmann method (LBM) has been applied to electrohydrodynamics (EHD) in recent years. In this paper, Shan-Chen (SC) single-component multiphase LBM is developed to study large-density-ratio EHD problems. The deformation/motion of a droplet suspended in a viscous liquid under an applied external electric field is studied with three different electric field models. The three models are leaky dielectric model, perfect dielectric model and constant surface charge model. They are used to investigate the effects of the electric field, electric properties of liquids and electric charges. The leaky dielectric model and the perfect dielectric model are validated by the comparison of LBM results with theoretical analysis and available numerical data. It shows that the SC LBM coupled with these electric field models is able to predict the droplet deformation under an external electric field. When net charges are present on the droplet surface and an electric field is applied, both droplet deformation and motion are reasonably predicted. The current numerical method may be an effective approach to analyze more complex EHD problems.
\end{abstract}

Keywords: Two-phase flows; lattice Boltzmann; electrohydrodynamics.

\section{Introduction}

There are many applications of electrohydrodynamics (EHD), such as spraying, inkjet printing and boiling. ${ }^{1}$ One of the most important research topics in EHD is a single drop's deformation/motion induced by an applied electric field. Under the influence of an applied electric field, a drop suspended in a viscous liquid may deform, move or burst. ${ }^{1-4}$ The deformation of a single drop suspended in another fluid under an electric field has been studied analytically using different models, ${ }^{1,3,4}$ such as the well-known leaky dielectric model which was proposed by Taylor. ${ }^{4}$

To study fluid flow in multiphase fluids, many theoretical and numerical studies were also carried out on this EHD problem. ${ }^{1,3-5}$ Usually the finite-element method $^{5}$ or the front tracking/finite-volume $\operatorname{method}^{6}$ was used to simulate the drop 
deformation in another fluid and the prediction on the small deformation of the drop agrees well with the asymptotic results. ${ }^{3,7}$

Recently, lattice Boltzmann method (LBM) has become a useful numerical tool in the study of fluid flows including the multiphase flow. ${ }^{8-10}$ Compared with conventional methods for multiphase flows, LBM is based on mesoscopic kinetic equations and usually the Poisson equation is not required to solve it. There are several popular multiphase models in LBM. ${ }^{11-14}$ Some large-density-ratio multiphase models ${ }^{10,11,14}$ have been successfully applied to study two-phase flow problems, such as droplet breakup, ${ }^{10}$ bubble collision, bubble rising, ${ }^{11}$ etc. However, these large-density-ratio models ${ }^{11,14}$ are complex because there are two sets of particle distribution functions (PDF). The simplest large-density-ratio model is the modified single-component multiphase Shan-Chen (SC) model.${ }^{15}$ In the model, the equation of state (EOS) of the fluid is incorporated by potentials of nonlocal interactions and the density ratio up to 1000 can be achieved. The SC model is also a diffuse-interface model. It contains thermodynamic aspects and may have underlying free energy. ${ }^{16}$ Hence, the topological transitions in flow field, such as breakup and coalescence, are handled automatically. The pressure tensor $p_{\alpha \beta}$ of the SC model is ${ }^{16}$

$$
p_{\alpha \beta}=\left[\left(c_{s}^{2} \rho+\frac{1}{2} g c_{s}^{2} \psi^{2}\right)+\frac{1}{2} g c_{s}^{4}\left(\psi \nabla^{2} \psi+\frac{1}{2}|\nabla \psi|^{2}\right)\right] \delta_{\alpha \beta}-\frac{1}{2} g c_{s}^{4} \partial_{\alpha} \psi \partial_{\beta} \psi,
$$

where $\rho$ is the density of the mixture, and $\psi(\rho)$ and constants $g, c_{s}^{2}$ will be introduced later. The well-known form of the pressure tensor based on a free-energy functional is ${ }^{14}$

$$
p_{\alpha \beta}=\left[p-\kappa \rho \nabla^{2} \rho-\frac{1}{2} \kappa(\nabla \rho)^{2}\right] \delta_{\alpha \beta}+\kappa \partial_{\alpha} \rho \partial_{\beta} \rho,
$$

where $p$ is the thermodynamic pressure determined by a non-ideal gas EOS. We can see that if $p=c_{s}^{2} \rho+\left(c_{s}^{2} g / 2\right) \psi^{2}, \kappa=-(1 / 2) g c_{s}^{4}$ and $\psi \propto \rho$, the pressure tensor in the SC model is consistent with the well-known form. Compared to the finite difference schemes for solving Cahn-Hilliard and Navier-Stokes equations, the SC LBM is much simpler and more efficient.

Till today only very few LBMs are applied to study EHD problems. Zhang and Kwok $^{17}$ used two-component SC LBM (see Ref. 12) to study the drop deformation under an external electric field, but in their study the densities of the drop and its surrounding fluid are identical. Huang et al. ${ }^{18}$ used single-component multiphase LBM to study the drop deformation and burst in gas. In their study, the formula $\varepsilon_{r}=1+\eta(\varsigma \rho)+\eta(\varsigma \rho)^{6}$ is proposed to mimic the permittivity property near the interface, where $\varepsilon_{r}$ is the relative permittivity, and $\eta$ and $\varsigma$ are adjustable parameters. ${ }^{18}$ However, the theoretical base of the above formula is unknown. Besides that, the simulation results seem sensitive to the choice of the adjustable parameters.

Here to study the drop deformation in gas, the SC single-component multiphase LBM is used. Compared with the large-density-ratio models proposed by 
Inamuro et al. ${ }^{11}$ and Lee and Lin, ${ }^{14}$ the $\mathrm{SC}$ model ${ }^{12,15}$ is simpler and more efficient since only one set of PDF is used. In our study, the density ratio of the two-fluids is much higher than that in the study of Zhang and Kwok. ${ }^{17}$ The permittivity and conductivity properties near the interface ${ }^{17}$ will be modeled more physically than that in the study of Huang et al. ${ }^{18}$

In our study, not only the leaky dielectric model, but also the perfect dielectric and constant surface charge model ${ }^{6}$ are used to study the effects due to different electric fields, electric properties of liquids, and electric charges. The leaky dielectric model is used for drops without net charges but finite electrical conductivity. The perfect dielectric model is used for drops of electrically isolating liquid. The constant surface charge model ${ }^{6}$ is also incorporated to simulate the cases with net charges on the drop surface.

In this paper, the single-component multiphase SC LBM will be applied to study the large-density-ratio drop deformation in an external electric field. First, we will briefly introduce the SC single-component multiphase LBM and the three different electric field models. Then the numerical results are discussed.

\section{Method}

\subsection{Shan-and-Chen-type single-component multiphase LBM}

Here we implement the SC LBM (see Ref. 12) in two dimensions for a singlecomponent multiphase system. In the model, one distribution function is introduced for the fluid. The distribution function satisfies the following lattice Boltzmann equation:

$$
f_{a}\left(\mathbf{x}+\mathbf{e}_{a} \Delta t, t+\Delta t\right)=f_{a}(\mathbf{x}, t)-\frac{\Delta t}{\tau}\left(f_{a}(\mathbf{x}, t)-f_{a}^{\mathrm{eq}}(\mathbf{x}, t)\right)
$$

where $f_{a}(\mathbf{x}, t)$ is the density distribution function in the ath velocity direction and $\tau$ is a relaxation time which is related to the kinematic viscosity as $\nu=c_{s}^{2}(\tau-0.5 \Delta t)$. The equilibrium distribution function $f_{a}^{\mathrm{eq}}(\mathbf{x}, t)$ can be calculated as

$$
f_{a}^{\mathrm{eq}}(\mathbf{x}, t)=w_{a} \rho\left[1+\frac{\mathbf{e}_{a} \cdot \mathbf{u}^{\mathrm{eq}}}{c_{s}^{2}}+\frac{\left(\mathbf{e}_{a} \cdot \mathbf{u}^{\mathrm{eq}}\right)^{2}}{2 c_{s}^{4}}-\frac{\left(\mathbf{u}^{\mathrm{eq}}\right)^{2}}{2 c_{s}^{2}}\right] .
$$

In Eqs. (2) and (3), the $\mathbf{e}_{a}$ 's are the discrete velocities. For the D2Q9 model, they are given by

$$
\left[\mathbf{e}_{0}, \mathbf{e}_{1}, \mathbf{e}_{2}, \mathbf{e}_{3}, \mathbf{e}_{4}, \mathbf{e}_{5}, \mathbf{e}_{6}, \mathbf{e}_{7}, \mathbf{e}_{8}\right]=c \cdot\left[\begin{array}{ccccccccc}
0 & 1 & 0 & -1 & 0 & 1 & -1 & -1 & 1 \\
0 & 0 & 1 & 0 & -1 & 1 & 1 & -1 & -1
\end{array}\right] .
$$

In Eq. (3), for the D2Q9 model, $w_{a}=4 / 9(a=0), w_{a}=1 / 9(a=1,2,3,4)$, $w_{a}=1 / 36(a=5,6,7,8), c_{s}=c / \sqrt{3}$, where $c=\Delta x / \Delta t$ is the ratio of lattice spacing $\Delta x$ and time step $\Delta t$. Here, we define one lattice unit $(\Delta x)$ as 1 lu and one time step $(\Delta t)$ as 1 ts. In Eq. (3), $\rho$ is the density of the fluid, which can be obtained from $\rho=\sum_{a} f_{a}$. 
In the SC LBM, the effect of body force is incorporated by adding an acceleration into the velocity field. The macroscopic velocity $\mathbf{u}^{\mathrm{eq}}$ is given by

$$
\mathbf{u}^{\mathrm{eq}}=\mathbf{u}^{\prime}+\frac{\tau \mathbf{F}}{\rho},
$$

where $\mathbf{u}^{\prime}$ is the velocity defined as

$$
\mathbf{u}^{\prime}=\frac{\sum_{a} f_{a} \mathbf{e}_{a}}{\rho} .
$$

In Eq. (4), $\mathbf{F}=\mathbf{F}_{\text {int }}+\mathbf{F}_{\text {Ele }}$ is the force acting on the fluid, here including the interparticle force $\mathbf{F}_{\text {int }}$ and an external electric force $\mathbf{F}_{\text {Ele }}$. The actual whole fluid velocity $\mathbf{u}$ is defined as ${ }^{19}$

$$
\mathbf{u}=\mathbf{u}^{\prime}+\frac{\mathbf{F}}{2 \rho}
$$

which means the "fluid velocity" should be calculated correctly by averaging the momentum before and after the collision. ${ }^{19}$ The interparticle force is defined $\mathrm{as}^{20}$

$$
\mathbf{F}_{\text {int }}(\mathbf{x}, t)=-g \psi(\mathbf{x}, t) \sum_{a} w_{a} \psi\left(\mathbf{x}+\mathbf{e}_{a} \Delta t, t\right) \mathbf{e}_{a},
$$

where $g$ is a parameter that controls the strength of the interparticle force. For the EOS proposed by Shan and Chen, ${ }^{12} \psi(\rho)=\rho_{0}\left[1-\exp \left(-\rho / \rho_{0}\right)\right]$, where $\psi$ is an effective number density ${ }^{12}$ and $\rho_{0}$ is a constant.

Through the Taylor expansion as described in Appendix A in Ref. 16 and $-\partial_{j} p+\partial_{i}\left(c_{s}^{2} \rho\right)=F_{i}$, here $i, j$ means the $x$ or $y$ coordinates, we obtained the pressure $p$ as ${ }^{16}$

$$
p=c_{s}^{2} \rho+\frac{c_{s}^{2} g}{2} \psi^{2}
$$

According to Yuan and Schaefer, ${ }^{15}$ if the EOS of $p=p(\rho)$ is already known, we can use the formula

$$
\psi=\sqrt{\frac{2\left(p-c_{s}^{2} \rho\right)}{c_{s}^{2} g}},
$$

to incorporate different EOS into the SC LBM.

Several typical EOSs are available but the Carnahan-Starling-van der Waals equation is found to be able to achieve the highest density ratio. Hence we would use this EOS in our study. The EOS is

$$
p=\rho R T \frac{1+b \rho / 4+(b \rho / 4)^{2}-(b \rho / 4)^{3}}{1-b \rho / 4}-a \rho^{2},
$$

with $a=0.4963 R^{2} T_{c}^{2} / p_{c}, b=0.18727 R T_{c} / p_{c} . R$ is the gas constant and $T$ is the temperature. We set $a=1, b=4$ and $R=1$. The critical temperature is 
$T_{c}=0.3773 a / b R$. In our simulations $T=0.57 T_{c}$. At the temperature with $\tau=1$, the densities of liquid and gas are $\rho_{l}=0.4217$ and $\rho_{g}=0.000407$, respectively. The density ratio is about 1036. The interfacial surface tension $\gamma$ is about 0.025 in our simulations. The surface tension was calculated using Laplace law after the equilibrium state is obtained in LBM simulations.

Suppose the flow field is isothermal and the drop suspension system is incompressible, the following electric body forces per unit volume should be added into the SC model through Eq. (4) due to the existence of an applied external electric field ${ }^{1,4}$;

$$
\mathbf{F}_{\mathrm{Ele}}=-\frac{1}{2} \mathbf{E} \cdot \mathbf{E} \nabla \varepsilon+\rho^{e} \mathbf{E}+\frac{1}{2} \nabla\left[\rho\left(\frac{\partial \varepsilon}{\partial \rho}\right)_{T} \mathbf{E} \cdot \mathbf{E}\right] .
$$

In the equation, $\mathbf{E}$ is the strength of the external electric field, $\varepsilon$ is the absolute conductivity and $\rho^{e}$ is the charge density. On the right-hand side of the above equation, the first term originates from polarization stress and acts along the normal direction of the interface. The second term is the electrostatic force acting along the direction of the electric field. The electrorestriction force term $-(1 / 2) \rho(\partial \varepsilon / \partial \rho)_{T} \mathbf{E} \cdot \mathbf{E}$ is neglected in our study because the fluid system is assumed to be incompressible.

Hence, the electric forces can be simplified $\mathrm{as}^{1}$

$$
\mathbf{F}_{\text {Ele }}=-\frac{1}{2} \mathbf{E} \cdot \mathbf{E} \nabla \varepsilon+\rho^{e} \mathbf{E} .
$$

To calculate the electric forces in Eq. (12), the electric field strength $(E)$ and charge density $\rho^{e}$ would be obtained using three electric field models. The three models are leaky dielectric model, perfect dielectric model and constant surface charge model.

In EHD, the magnetic induction effects can be ignored and $\nabla \times \mathbf{E}=0 .{ }^{1,4}$ Hence, the $E$ can be written as $\mathbf{E}=-\nabla \phi$, where $\phi$ is the electric potential.

The Gauss law in a dielectric material with permittivity $\varepsilon$ is $\nabla \cdot(\varepsilon \mathbf{E})=\rho^{e}$, where the $\rho^{e}$ is the density of local free charges.

For all these three models, the governing equations are valid,

$$
\nabla \times \mathbf{E}=0, \quad \nabla \cdot(\varepsilon \mathbf{E})=\rho^{e} .
$$

The charge conservation equation is ${ }^{6}$

$$
\frac{D \rho^{e}}{D t}=\frac{\partial \rho^{e}}{\partial t}+\mathbf{u} \cdot \nabla \rho^{e}=-\nabla \cdot(\sigma \mathbf{E})
$$

where $\sigma$ is electrical conductivity. For the leaky dielectric model, Eq. (14) can be simplified as

$$
\nabla \cdot(\sigma \mathbf{E})=0,
$$

and furthermore, the equation can be written as $\nabla \cdot(\sigma \nabla \phi)=0$ (see Ref. 6). After solving this equation, the electric field strength is calculated by $\mathbf{E}=-\nabla \phi$. On the other hand, one can obtain the distribution of charge density using $\rho^{e}=\nabla \cdot(\varepsilon \mathbf{E})$. 
For the perfect dielectric model, there is no free charge in the medium, i.e. $\rho^{e}=0$ (see Ref. 6). Hence, from Eq. (13), one can obtain that $\nabla \cdot(\varepsilon \nabla \phi)=0$. For given electric potentials in the boundary, this governing equation for electric potential can be solved. Hence, the electric field strength $\mathbf{E}$ can be obtained through $\mathbf{E}=-\nabla \phi$. With the condition of no free charge $\rho^{e}=0$, Eq. (12) can be simplified as, $\mathbf{F}_{\text {Ele }}=-(1 / 2) \mathbf{E} \cdot \mathbf{E} \nabla \varepsilon$.

For the constant surface charge model, the free charges are assumed to be distributed uniformly on the drop interface and the total charge is kept constant. The assumption is valid when the applied electric field strength is not strong and the charge density is low. ${ }^{6}$

The surface charge density $q^{s}$ satisfies

$$
q^{s}=\int_{-l}^{l} \rho^{e} d l, \quad \nabla \cdot(\varepsilon \mathbf{E})=\rho^{e} .
$$

where $l$ means that the free-charge distribution spans on both sides of the interface.

Solving the equation

$$
\nabla \cdot(\varepsilon \nabla \phi)=\rho^{e}
$$

with electric potentials on the boundary, we can obtain the local electric strength $\mathbf{E}$. Then, the surface charge density would be obtained as $\rho^{e}=\nabla \cdot(\varepsilon \mathbf{E})$.

Due to the non-uniform electric conductivity, permittivity and electric field, and free charges in the interface, the drop motion is affected by the applied electric field. ${ }^{17}$ Using the density of fluid as an index function, the dielectric properties can be expressed as

$$
\varepsilon(\rho)=\frac{\rho-\rho_{i}}{\rho_{e}-\rho_{i}} \varepsilon_{e}+\frac{\rho-\rho_{e}}{\rho_{i}-\rho_{e}} \varepsilon_{i}, \quad \sigma(\rho)=\frac{\rho-\rho_{i}}{\rho_{e}-\rho_{i}} \sigma_{e}+\frac{\rho-\rho_{e}}{\rho_{i}-\rho_{e}} \sigma_{i},
$$

where $\varepsilon_{i}$ and $\varepsilon_{e}$ are the permittivity constants for the fluid inside and outside of the drop, and $\sigma_{i}$ and $\sigma_{e}$ are the conductivity constants of the two fluids. $\rho_{i}$ and $\rho_{e}$ are the densities of the fluid inside and outside the drop. Using Eq. (18), the variation of dielectric properties near the interface is smooth and does not affect the original interface thickness.

For the constant surface charge model, the free charge density is set to be proportional to the absolute density gradient, ${ }^{6}$ i.e. $\rho^{e} \propto|\nabla \rho|$. In the constant surface charge model, the charge conservation equation is $Q=\Sigma \rho^{e}$, where $Q$ is the total charges on the drop's surface. Hence, the surface charge density is

$$
\rho^{e}(\rho)=\frac{Q}{\Sigma|\nabla \rho|}|\nabla \rho|
$$

In the three models, the following Laplace's system governs the electric field,

$$
\nabla \cdot(k \nabla \phi)=q .
$$


Here for simplicity, the alternating direction implicit (ADI) iteration was used to solve the Laplace's system. For the iteration in each particular direction, the efficient tri-diagonal matrix algorithm (TDMA) is used.

\section{Results and Discussion}

In our study, the external electric field vector $\mathbf{E}_{0}$ is along the $Y$ direction. In the following discussion, $D$ is the deformation factor defined as $D=(L+B) /(L-B)$, where $L$ is the end-to-end length of the droplet measured along the direction of electric field ( $Y$ axis) and $B$ is the maximum breadth in the traverse direction $(X$ axis). Positive and negative $D$ 's mean the drop increases in length in the $Y$ axis (prolate) and $X$ axis (oblate), respectively.

An electrical capillary number $C a_{E}$ or Weber number $W e$, defined as $C a_{E}=W e=\varepsilon_{e} R_{0} E_{0}^{2} / \gamma$, is introduced here to illustrate the dimensionless strength of the external electric field $E_{0}$ compared to the interfacial tension constant $\gamma$. In our system, the external electric field strength $E_{0}$ can be calculated by dividing the potential difference between the two parallel electrode walls by the distance in the $Y$ direction.

\subsection{Leaky dielectric model}

Using the leaky dielectric model, the asymptotic solution with a first-order approximation for two-dimensional (2D) drop deformation in the electric field is ${ }^{7}$

$$
D=\frac{R_{0} \varepsilon_{e} E_{0}^{2} d}{3 \gamma(1+H)^{2}}=\frac{d W e}{3(1+H)^{2}},
$$

where the discriminating function $d=H^{2}+H+1-3 S, H=\sigma_{i} / \sigma_{e}$ and $S=\varepsilon_{i} / \varepsilon_{e}$. This 2D deformation first-order solution seems similar to that of the three-dimensional (3D) one in Ref. 4 . The difference is that the discriminating function in $3 \mathrm{D}$ is not only the function of $H$ and $S$, but also the viscosity ratio of the two fluids.

In the LBM simulation, the density ratio between drop and surrounding gas is 1036. The computational domain is $201 \times 201$; the potentials in the upper and lower boundaries are specified as $\phi^{+}=50$ and $\phi^{-}=0$, respectively, so that $E_{0}=$ $\left(\phi^{+}-\phi^{-}\right) / 200=0.25$. The initial drop radius is $23 \mathrm{lu}$. The final steady deformation factor $D$ for nine cases with different conductivity and permittivity constants are illustrated in Table 1. The asymptotic solution is also shown in the table. From the table, it is found that for the discriminating function $d<0$, the drop becomes prolate $(D>0)$ and for $d>0$, the drop becomes oblate $(D<0)$. From Fig. 1 and Table 1 , we can see that for the small deformation $(D<0.1)$, the LBM results agree well with the asymptotic solutions. When the deformation is large $(D>0.1)$, the asymptotic solution underestimates the deformation, which is highly consistent with the conclusions in other numerical studies. ${ }^{1,5}$

When the deformation of a leaky dielectric drop becomes steady under the applied electric field, vortices would be formed both inside and outside of the drop. 
Table 1. The discriminating function $d$ and the deformation factor $D$ for different combinations of the conductivity and permittivity. (In the LBM simulations $E_{0}=0.25$ with mesh $201 \times 201$ and density-ratio is about 1036).

\begin{tabular}{ccccccr}
\hline$\varepsilon_{i}$ & $\varepsilon_{e}$ & $\sigma_{i}$ & $\sigma_{e}$ & $d$ & $D(\mathrm{LBM})$ & $D$ [Eq. (21)] \\
\hline 0.020 & 0.010 & 0.10 & 0.05 & 1.0 & 0.02222 & 0.02337 \\
0.010 & 0.010 & 0.20 & 0.10 & 4.0 & 0.08696 & 0.09347 \\
0.010 & 0.020 & 0.10 & 0.10 & 1.5 & 0.14894 & 0.14680 \\
0.010 & 0.020 & 0.10 & 0.05 & 5.5 & 0.33333 & 0.25705 \\
0.005 & 0.010 & 0.10 & 0.05 & 5.5 & 0.13043 & 0.12853 \\
0.015 & 0.010 & 0.10 & 0.10 & -1.5 & -0.08696 & -0.07887 \\
0.010 & 0.010 & 0.10 & 0.20 & -1.25 & -0.13043 & -0.11684 \\
0.020 & 0.010 & 0.10 & 0.10 & -3.0 & -0.14894 & -0.14680 \\
0.018 & 0.020 & 0.05 & 0.10 & -1.0 & -0.21739 & -0.17760 \\
\hline
\end{tabular}

The vortex directions are determined only by the sign of $(H-S)$ and are not relevant to the other parameters, for example, the discriminating function $d .^{1,5,6}$ When $H>S$, from the definition of discriminating function, we can see that obviously $d>0$. But when $H<S$, the discriminating function can be positive or negative. Figure 2 shows the flow patterns for the following three typical cases: (a) $H>S, d>0$; (b) $H<S, d>0$ and (c) $H<S, d<0$.

In the simulations, the density ratio is $1036: 1$. In each panel of Fig. 2, only a quarter of the central computational domain is illustrated due to symmetry. For the case (a), $E_{0}=0.25, \varepsilon_{i}=0.005, \varepsilon_{e}=0.01, \sigma_{i}=0.1$ and $\sigma_{e}=0.05$, the Taylor vortex inside the drop is counterclockwise. For the case (b), $E_{0}=0.25, \varepsilon_{i}=0.02, \varepsilon_{e}=$ $0.01, \sigma_{i}=0.1$ and $\sigma_{e}=0.1$, the Taylor vortex inside the drop is clockwise. For the case (c), $E_{0}=0.13, \varepsilon_{i}=0.018, \varepsilon_{e}=0.02, \sigma_{i}=0.05$ and $\sigma_{e}=0.1$, the Taylor vortex

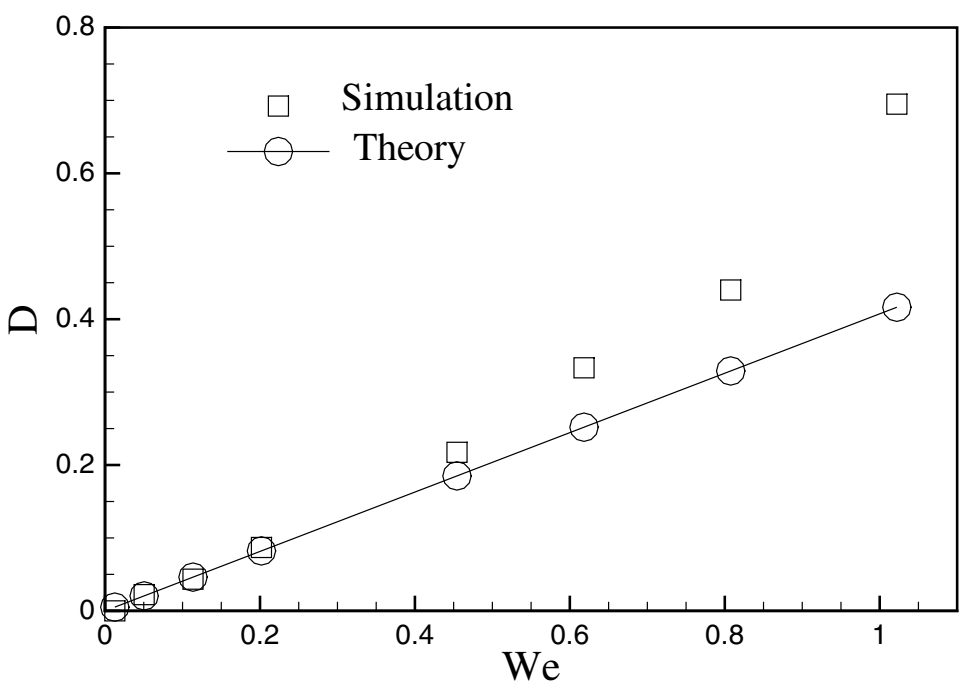

Fig. 1. Deformation factor $D$ as a function of $W e$. In the simulation, $H=2$ and $S=0.5$. 


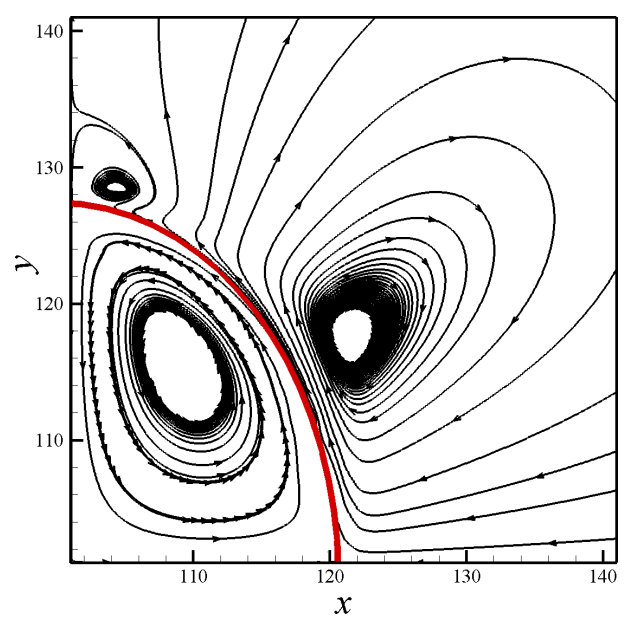

(a)

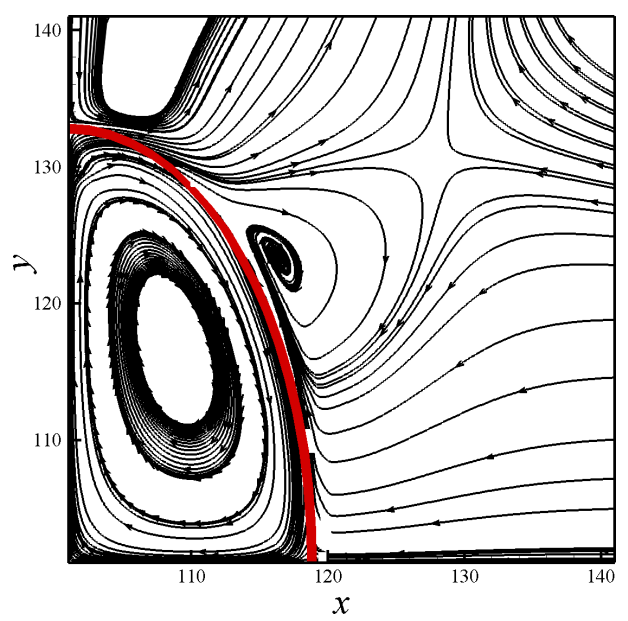

(b)

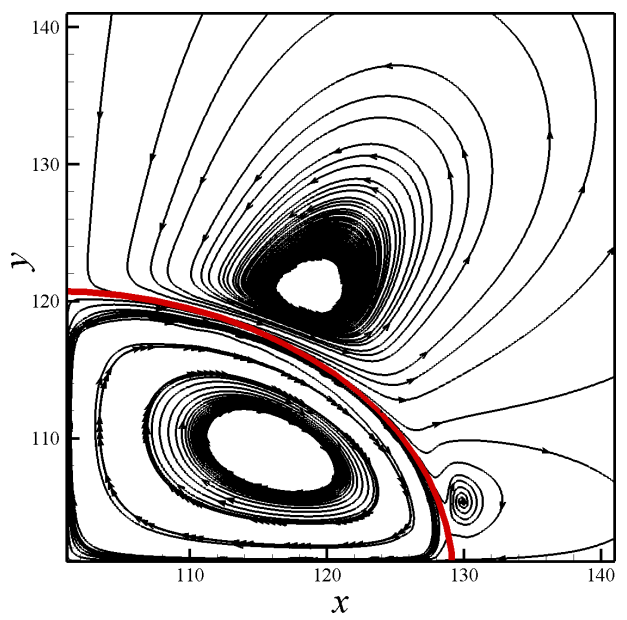

(c)

Fig. 2. (Color online) Flow patterns for three typical cases: (a) $H>S, d>0$; (b) $H<S, d>0$; (c) $H<S, d<0$. Only a quarter of the central computational domain is illustrated.

inside the drop is also clockwise. These results are consistent with the other numerical results. ${ }^{6,17}$ Here we can see that, although the signs of $d$ are different in case (b) and (c), the vortex directions are same. The vortex directions for case (a), and cases (b) and (c) are different because the sign of $(H-S)$ in case (a) is positive while in cases (b) and (c) it is negative. , $^{1,5}$

\subsection{Perfect dielectric model}

When the electrical conductivity of the system is too low, the perfect dielectric model should be used to carry out simulations. In this section, firstly the asymptotic 
Table 2. The comparison of the numerical and asymptotic solution for deformation of a perfect dielectric drop $(D)$ in the electric field.

\begin{tabular}{ccccc}
\hline$\varepsilon_{i}$ & $\varepsilon_{e}$ & $E_{0}$ & $D(\mathrm{LBM})$ & $D$ (asymptotic [Eq. (A.10)]) \\
\hline 0.20 & 0.10 & 0.20 & 0.15556 & 0.14956 \\
0.40 & 0.20 & 0.10 & 0.15556 & 0.14951 \\
0.70 & 0.20 & 0.10 & 0.26087 & 0.20772 \\
0.25 & 0.20 & 0.15 & 0.02222 & 0.01869 \\
0.10 & 0.20 & 0.10 & 0.06667 & 0.07478 \\
0.10 & 0.40 & 0.10 & 0.29167 & 0.48456 \\
\hline
\end{tabular}

solution for the deformation of a perfect dielectric drop in an electric field (refer to the Appendix) was derived. Then the LBM simulations were carried out. Table 2 shows the deformation solution obtained by LBM. The asymptotic solutions obtained by Eq. (A.10) are also illustrated. In Table 2, it is found that when $D<0.1$, the LBM results agree well with the asymptotic solutions. Figure 3 further shows the comparison of theoretical predictions and results of LBM simulations for cases $S>1$ and $S<1$. We can see that when the $S>1$ and $D$ is small, the LBM results are consistent with the predictions. However, if $S>1$ and $D$ is larger, the theoretical predictions underestimate the $D$ value. On the other hand, from Fig. 3(b), we can see that if $S<1$ and $D$ is large, the theoretical predictions overestimate the $D$ values. All these results are highly consistent with the numerical results in Ref. 21 .

In general, the perfect dielectric drop deforms into a prolate shape. ${ }^{6}$ Figure 4 shows a typical flow pattern in a perfect dielectric drop under an electric field. It shows the state when the drop is stable. In the simulation, $E_{0}=0.09, \varepsilon_{i}=0.7$ and $\varepsilon_{e}=0.2$.

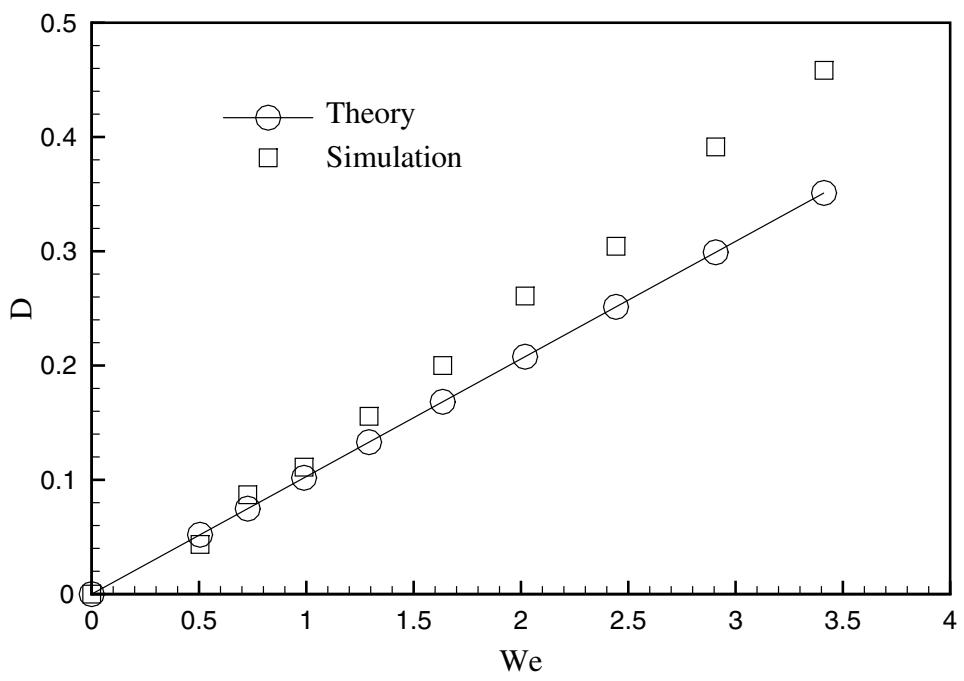

(a)

Fig. 3. (a) $S>1\left(\varepsilon_{i}=0.7\right.$ and $\left.\varepsilon_{e}=0.2\right)$; (b) $S<1\left(\varepsilon_{i}=0.1\right.$ and $\left.\varepsilon_{e}=0.4\right)$. 


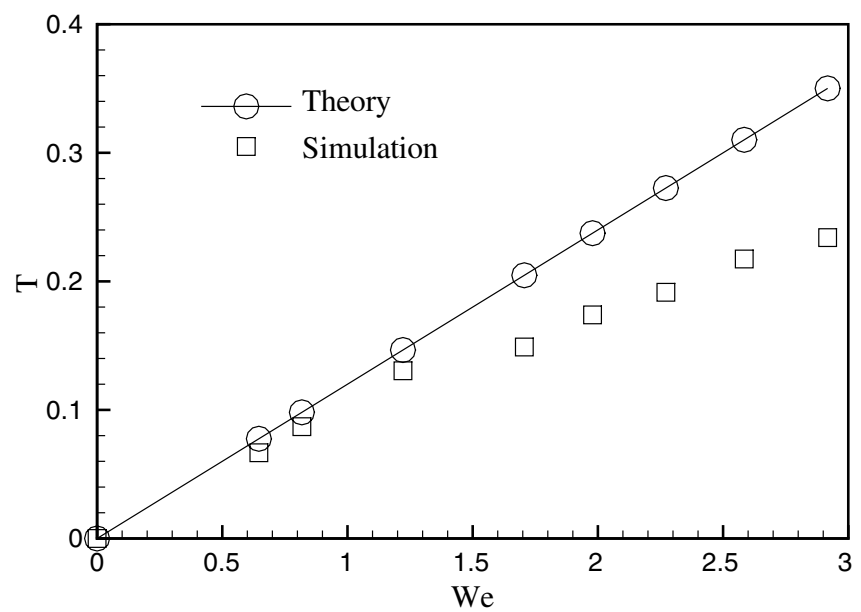

(b)

Fig. 3. (Continued)

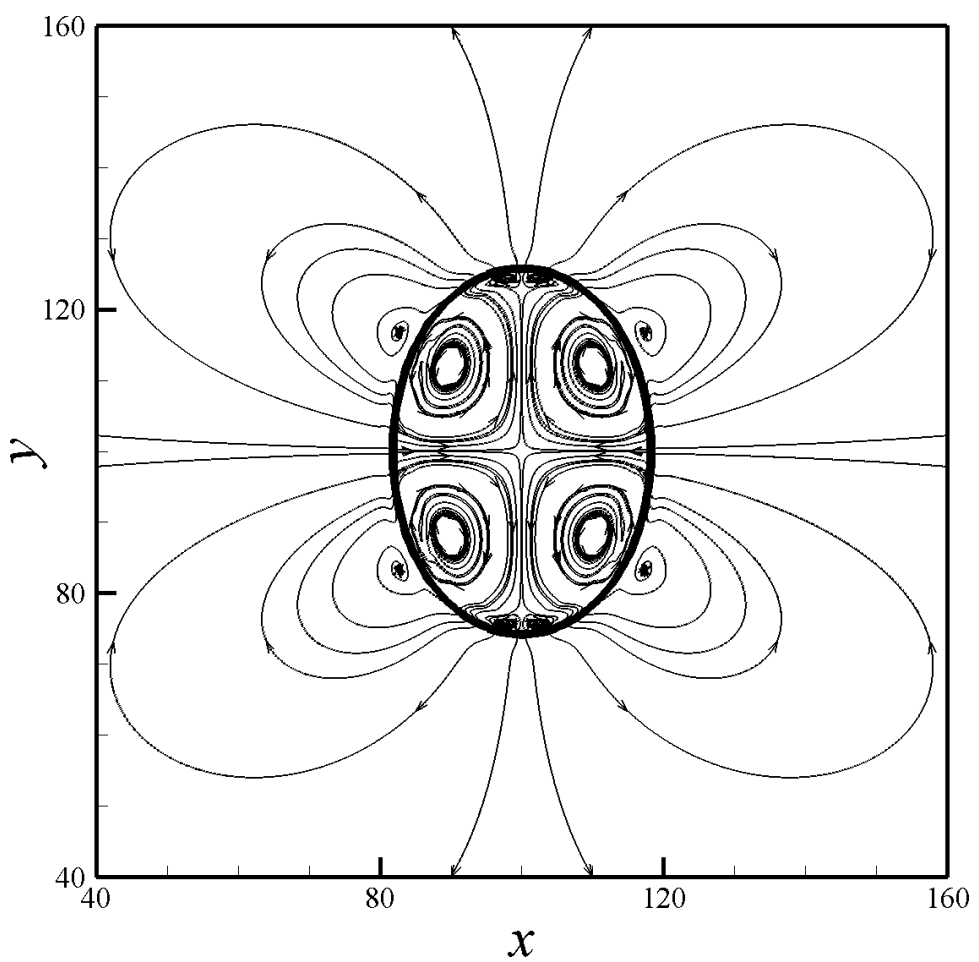

Fig. 4. A typical flow pattern in a perfect dielectric drop under an electric field with $E_{0}=0.09, \varepsilon_{i}=0.7$ and $\varepsilon_{e}=0.2$. 
Compared to the strong vortices inside the leaky dielectric drop, the vortices inside the perfect dielectric drop are much weaker. Besides, in the two ends of the prolate drop, four very weak Taylor vortices are generated.

As we know, in the equilibrium state, there is no fluid flow inside a perfect dielectric drop. ${ }^{6}$ It is noted in Fig. 4 that there are some weak flow patterns. This may be attributed to the following two factors: (a) the final drop shape is obtained from the initial shape of a sphere. During our transient simulation, the induced flows would decay with time but the time for the vortices to vanish is much longer than that for the drop shape to become stable ${ }^{6}$; (b) in LBM simulations, usually a spurious velocity is induced near the interface although the magnitude is very small.

\subsection{Constant surface charge model}

For the above leaky and perfect dielectric drop deformation under an electric field, the drop center is not moved. However, when there are net charges on the drop surface, because of the electrostatic force, the suspended drop will be moved. ${ }^{6}$

Figure 5 shows the deformation and motion of a charged drop under an electric field using the constant surface charge model. In the figure, the drop deforms into a prolate shape and moves along the electric field.

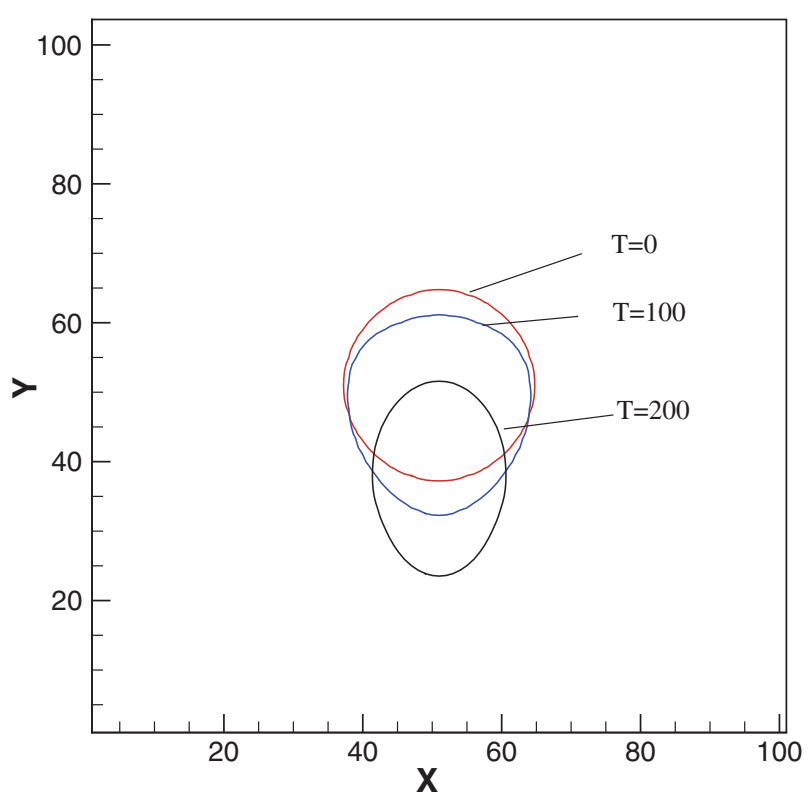

(a)

Fig. 5. (Color online) Deformation and motion of a droplet with constant charges in the surface under an electric field. In the simulation, computational domain is $101 \times 101$ and initial radius of the drop is $11 l u$. The other parameters are $E_{0}=0.1, \varepsilon_{i}=0.4$ and $\varepsilon_{e}=0.2$. 


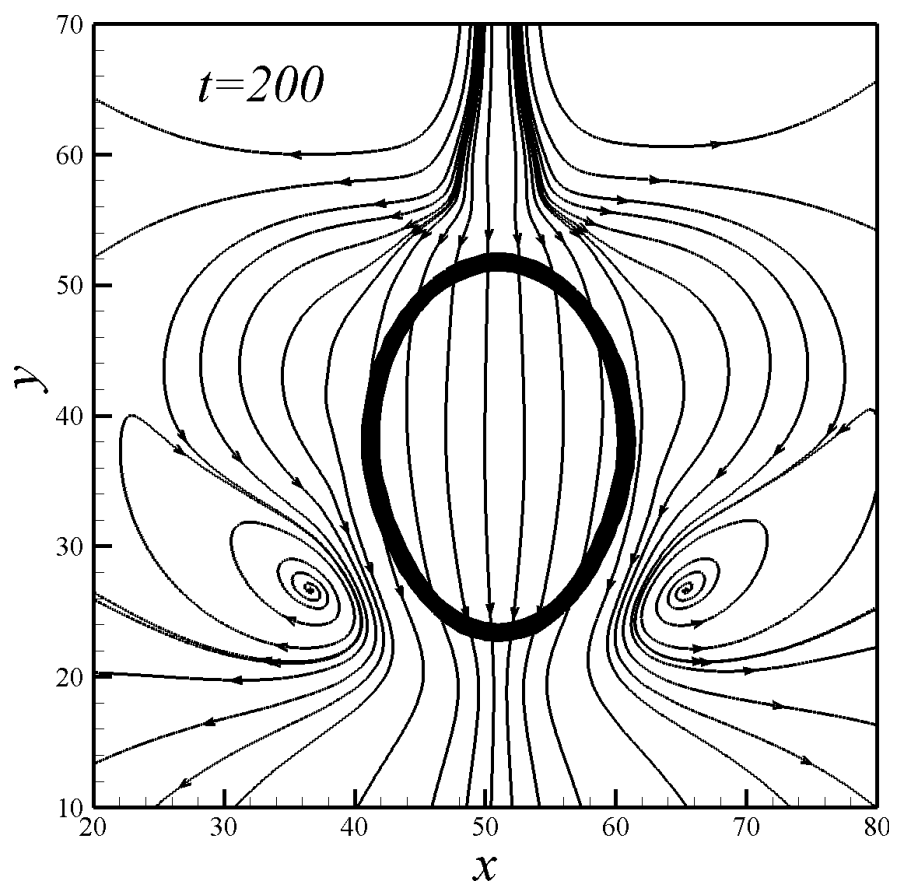

(b)

Fig. 5. (Continued)

The flow pattern at time $T=200 \mathrm{ts}$ shows that the moving liquid makes the surrounding fluid move, resulting in two circulating vortices outside the droplet.

\section{Conclusion}

The SC single-component multiphase LBM was developed to study large-densityratio EHD problems. The deformation/motion of a droplet suspended in a viscous liquid under an applied external electric field is studied with leaky dielectric, perfect dielectric and constant surface charge models. Our results are highly consistent with the available asymptotic and numerical results. ${ }^{5,6,17}$ This study demonstrates that SC single-component multiphase LBM is a very good tool to study the droplet deformation and motion under an external electric field due to its simplicity and capability of achieving large density ratio.

\section{Acknowledgments}

This work was supported by the Fundamental Research Funds for the Central Universities of China. 


\section{Appendix. Deformation of a Perfect Dielectric Drop in an Electric Field}

Due to the force balance between the electric stress developed on the interface and the interfacial tension, an incompressible fluid drop in an electric field may deform. ${ }^{2}$ Here we would discuss the case of a perfect dielectric drop in another dielectric fluid. The following analysis is limited to $2 \mathrm{D}$ small deformations.

The potentials inside and outside of a circle of radius $R_{0}$ are $\phi_{i}$ and $\phi_{e}$, respectively. The dielectric drop is in a macroscopically uniform electric field of strength $E$ directed along the $Y$ axis of a Cartesian co-ordinate system. The potentials are given by the following electrostatic equations ${ }^{2}$ :

$$
\begin{gathered}
\phi_{i}=-\frac{2}{S+2} E_{0} r \cos \theta \\
\phi_{e}=-\left[1-\frac{R_{0}^{2}}{r^{2}}\left(\frac{S-1}{S+1}\right)\right] E_{0} r \cos \theta
\end{gathered}
$$

where $(r, \theta)$ are the polar co-ordinates with the drop centered at the origin, $\theta$ is the angle between the radius vector and the $Y$ axis, and $S$ is the ratio of the dielectric constant of the drop $\left(\varepsilon_{i}\right)$ to that of the surrounding medium $\left(\varepsilon_{e}\right)$. The tangential and normal components of the local field strengths on the outside of the drop are

$$
\begin{aligned}
& E_{t, e}=-\frac{1}{R_{0}}\left(\frac{\partial \phi_{e}}{\partial \theta}\right)_{r=R_{0}}=-\frac{2}{S+1} E_{0} \sin \theta, \\
& E_{n, e}=-\left(\frac{\partial \phi_{e}}{\partial r}\right)_{r=R_{0}}=\frac{2 S}{S+1} E_{0} \cos \theta
\end{aligned}
$$

The normal electric stress $\left(f_{n}\right)$ acting outwards on the interface is (Smythe, 1953)

$$
f_{n}=\frac{\varepsilon_{i}-\varepsilon_{e}}{2 \varepsilon_{e}}\left[\frac{\left(\varepsilon_{e} E_{t, e}\right)^{2}}{\varepsilon_{e}}+\frac{\left(\varepsilon_{e} E_{n, e}\right)^{2}}{\varepsilon_{i}}\right] .
$$

Simplifying the above equation, one can obtain

$$
f_{n}=\frac{\varepsilon_{e} E_{0}^{2}(S-1)^{2}}{(S+1)^{2}}(\mu+\cos 2 \theta), \quad \mu=\frac{\varepsilon_{i}+\varepsilon_{e}}{\varepsilon_{i}-\varepsilon_{e}}=\frac{S+1}{S-1} .
$$

To balance the electric stress $\left(f_{n}\right)$, the $2 \mathrm{D}$ drop's curvature must satisfy the Laplace law,

$$
\frac{\gamma}{R_{c}}=\left(p_{i}-p_{e}\right)+f_{n}
$$

where $R_{c}$ is the radius of curvature at any point on the interface, $p_{i}$ and $p_{e}$ are the hydrostatic pressures inside and outside of the drop, respectively, and $\gamma$ is the interfacial tension. According to the asymptotic analysis, ${ }^{22}$ we suppose that the 
drop's shape satisfy the following equation of an ellipse:

$$
r=R_{0}\left[1+\frac{2}{3} D\left(3 \cos ^{2} \theta-1\right)\right]
$$

where $D$ is the deformation factor. Hence, the curvature of the ellipse is:

$$
\begin{aligned}
\frac{1}{R_{c}}= & 1-\frac{D}{3}-\frac{43 D^{2}}{18}+\frac{133 D^{2}}{54}+\frac{D}{4}\left(12-8 D+7 D^{2}\right) \cos 2 \theta \\
& +\frac{9}{2}(D-1) D^{2} \cos 4 \theta+\frac{41}{4} D^{3} \cos 6 \theta
\end{aligned}
$$

Then we substitute Eqs. (A.9) and (A.6) into that of Eq. (A.7). Supposing D satisfies the assumption of small deformation, series expansion around zero can be performed. Omitting the terms higher than first order and comparing the coefficient before the term $\cos (2 \theta)$, one can obtain

$$
D=\frac{R_{0} \varepsilon_{e} E_{0}^{2}}{\gamma} \frac{(1-S)^{2}}{3(1+S)^{2}}=\frac{(1-S)^{2}}{3(1+S)^{2}} W e .
$$

This is the asymptotic solution of the deformation of a perfect dielectric drop in the surrounding dielectric medium under an electric field.

It is noted that if the $S$ value in Eq. (A.10) is $S=\infty$, then

$$
D=\frac{R_{0} \varepsilon_{e} E_{0}^{2}}{3 \gamma}=\frac{W e}{3}
$$

This is the solution of an electric drop in the surrounding dielectric medium under an electric field.

In what follows, let us compare our explicit formula [Eq. (A.10)] with an implicit formula proposed by Miksis. ${ }^{23}$ Miksis studied the effect of the critical $S_{c}$ (ratio of dielectric constant) on the deformation of the drop and the implicit solution for the 2 D drop-deformation for $S>1$ was given $\mathrm{as}^{23}$

$$
E_{0} \sqrt{4 \pi \varepsilon_{e} \frac{R_{0}}{\gamma}} \approx \sqrt{8 \pi}\left(\frac{1}{S-1}+\frac{1}{1+x}\right)\left(x^{3 / 2}+x^{-3 / 2}\right)^{1 / 2}, \quad x=\frac{1+D}{1-D} .
$$

Notice that the minor differences between the above equation and the original one ${ }^{23}$ are due to the international system of units used here and that the coefficient $\sqrt{8 \pi}$ is omitted from the original one. The critical dielectric-constant-ratio is shown as $S=50.73$ (see Ref. 23). Hence in the study of Huang et al., ${ }^{18}$ the conclusion of $14 \leq S_{c} \leq 15$ is questionable.

Squaring the above equation and performing series expansion around zero, one can obtain

$$
\frac{R_{0} \varepsilon_{e} E_{0}^{2}}{\gamma} \approx \frac{3(1+S)^{2}}{(1-S)^{2}} D+\frac{3(1+S)^{2}}{(1-S)^{2}} D^{2}+O\left(D^{3}\right)
$$


Omitting the terms higher than $O\left(D^{1}\right)$, the explicit solution of $D$ is identical as that in Eq. (A.10), but the explicit asymptotic solution of a 2D dielectric dropdeformation [Eq. (A.10)] is more general because when $S<1$, it is still valid.

\section{References}

1. D. A. Saville, Annu. Rev. Fluid Mech. 29, 27 (1997).

2. R. S. Allan and S. G. Mason, Proc. R. Soc. London, Ser. A 267, 45 (1962).

3. G. I. Taylor, Proc. R. Soc. London, Ser. A 291, 159 (1966).

4. J. R. Melcher and G. I. Taylor, Annu. Rev. Fluid Mech. 1, 111 (1969).

5. J. Q. Feng and T. C. Scott, J. Fluid Mech. 311, 289 (1996).

6. J. Hua, L. K. Lim and C. Wang, Phys. Fluids 20, 113302 (2008).

7. J. Q. Feng, J. Colloid Interface Sci. 246, 112 (2002).

8. A. Kuzmin, A. A. Mohamad and S. Succi, Int. J. Mod. Phys. C 19, 875 (2008).

9. J. J. Huang, C. Shu, Y. T. Chew and H. W. Zheng, Int. J. Mod. Phys. C 18, 492 (2007).

10. D. Chiappini, G. Bella, S. Succi and S. Ubertini, Int. J. Mod. Phys. C 20, 1803 (2009).

11. T. Inamuro, T. Ogata, S. Tajima and N. Konishi, J. Comput. Phys. 198, 628 (2004).

12. X. Shan and H. Chen, Phys. Rev. E 47, 1815 (1993).

13. D. J. Holdych, D. Rovas, J. G. Georgiadis and R. O. Buckius, Int. J. Mod. Phys. C 9, 1393 (1998).

14. T. Lee and C. L. Lin, J. Comput. Phys. 206, 16 (2005).

15. P. Yuan and L. Schaefer, Phys. Fluids 18, 042101 (2006).

16. X. Shan and H. Chen, Phys. Rev. E 49, 2941 (1994).

17. J. Zhang and D. Y. Kwok, J. Comput. Phys. 206, 105 (2005).

18. W. Huang, Y. Li and Q. Liu, Chin. Sci. Bull. 52, 3319 (2007).

19. X. Shan and G. Doolen, J. Stat. Phys. 81, 379 (1995).

20. N. S. Martys and H. D. Chen, Phys. Rev. E 53, 743 (1996).

21. E. Lac and G. M. Homsy, J. Fluid Mech. 590, 239 (2007).

22. C. S. Park, N. A. Clark and R. D. Noble, Phys. Rev. Lett. 72, 1838 (1994).

23. M. J. Miksis, Phys. Fluids 24, 1967 (1981). 